\title{
Hydrodynamic Instability Experiments and Simulations
}

\author{
Guy Dimonte, Marilyn Schneider and C. Eric Frerking
}

5th International Workshop on the Physics of Compressible Turbulent Mixing, July 18-21 1995, Stony Brook, New York

July, 1995

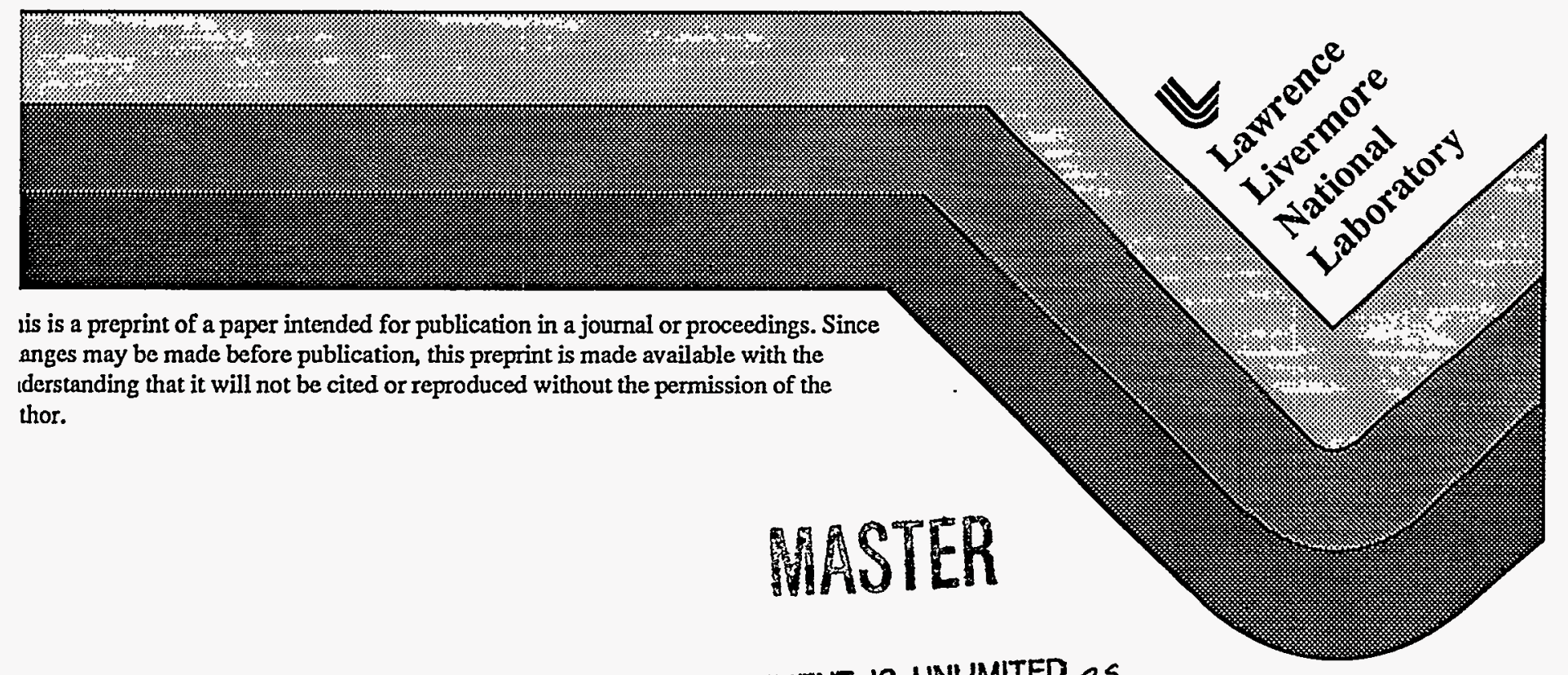

DISTRIBUTION OF THIS DOCUMENT IS UNLUMITED 85 


\section{DISCLAIMER}

This document was prepared as an account of work sponsored by an agency of the United States Government. Neither the United States Government nor the University of California nor any of their employees, makes any warranty, express or implied, or assumes any legal liability or responsibility for the accuracy, completeness, or usefulness of any information, apparatus, product, or process disclosed, or represents that its use would not infringe privately owned rights. Reference herein to any specific commercial products, process, or service by trade name, trademark, manufacturer, or otherwise, does not necessarily constitute or imply its endorsement, recommendation, or favoring by the United States Government or the University of California. The views and opinions of authors expressed herein do not necessarily state or reflect those of the United States Government or the University of California, and shall not be used for advertising or product endorsement purposes. 


\section{DISCLAIMER}

Portions of this document may be illegible in electronic image products. Images are produced from the best available original document. 


\title{
Hydrodynamic Instability Experiments and Simulations
}

\author{
Guy Dimonte, Marilyn Schneider, and C. Eric Frerking \\ Lawrence Livermore National Laboratory \\ Livermore, California 94551
}

Richtmyer-Meshkov experiments are conducted on the Nova laser with strong radiatively driven shocks (Mach $>20$ ) in planar, two-fluid targets with Atwood number A $<0$. Single mode interfacial perturbations are used to test linear theory and 3D random perturbations are used to study turbulent mix. Rayleigh-Taylor experiments are conducted on a new facility called the Linear Electric Motor (LEM) in which macroscopic fluids are accelerated electro-magnetically with arbitrary acceleration profiles. The initial experiments are described. Hydrodynamic simulations in 2D are in reasonable agreement with the experiments, but these studies show that simulations in 3D with good radiation transport and equation of state are needed.

\section{Introduction}

The Richtmyer-Meshkov (RM) [1,2] and Rayleigh-Taylor (RT) [3] instabilities occur when fluids of different densities are accelerated. In the RM instability, the acceleration is impulsive from a shock and the fluid compression is important. In the RT instability, the acceleration is sustained but will vary in time in applications like inertial confinement fusion [4]. We investigate both instablities using different experimental facilities and simulations using a Lagrangian-Eulerian code, CALE [5].

The RM experiments [6-8] are performed on the Nova laser at LLNL where pressures exceeding $30 \mathrm{Mb}$ are obtained. with a radiation drive. This extends. previous investigations in shock tubes $[2,9,10]$ to very high compression. We also use experimental techniques that avoid membranes and edge effects that have plagued shock tube experiments [11]. Experiments are conducted with single sinusoidal perturbations at the interface to test linear theory. We find that the linear growth rates agree with a model suggested by Meyer and Blewett [12]. We also studied compressible turbulent mix with 3D random interfacial pertubations. The mix width is found to increase in time as $h \sim t_{t} \beta$ with $\beta \sim 0.6 \pm 0.1$. Hydrodynamic simulations in $2 \mathrm{D}$ with a Lagrangian-Eulerian code [5] obtain comparable amplitudes but the exponent is larger, $\beta \sim 0.9$.

The RT instability is investigated using a linear electric motor (LEM) to accelerate macroscopic fluids similar to the "rocket rig" experiments [13]. The acceleration is electro-magnetic so that the temporal shape is adjustable to generalize the mix scaling laws [13]. We obtain a constant acceleration $\sim 70 \mathrm{~g}_{\mathrm{o}}$ (earth's gravity) or an impulsive acceleration with a peak $\sim 700 \mathrm{~g}_{0}$. The initial results are reported here.

\section{RM experiments on Nova}

The Nova experimental configuration has been described previously [6-8] and is shown in Fig. 1. In order to produce a spatially uniform drive, eight laser beams $(28 \mathrm{~kJ}$ at $0.53 \mu \mathrm{m})$ are converted to soft $x$-rays inside a cylindrical radiation enclosure called a hohlraum ( 2-3 mm diameter, 3-4 mm length). A 3 ns laser pulse generates a quasi- 
Plankian x-ray drive with a peak radiation temperature of $\sim 140 \mathrm{eV}$. A HIGH and LOW drive is used in our experiments with different size hohlraums. The target is mounted on the hohlraum wall and radiographed in-flight from the side and face-on using $x$-rays generated by two independent laser beams. A gated $x$-ray imager (GXI) obtains 2D radiographs and an $x$-ray streak camera obtains $1 D$ images streaked in time.

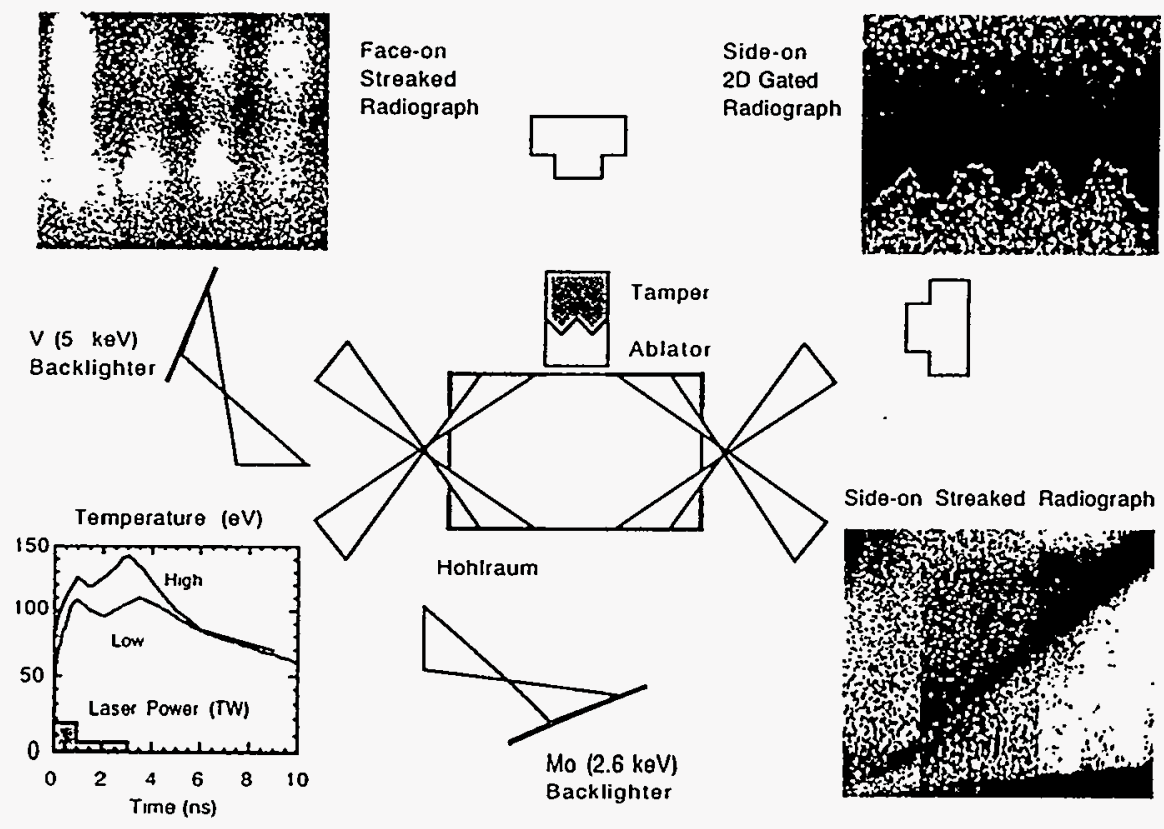

Figure 1: Experimental configuration and sample data

The target consists of a berylium (Be) ablator with density $\rho_{1} \sim 1.7 \mathrm{~g} / \mathrm{cm}^{3}$ and a foam tamper $\left(\rho_{2}=0.12 \mathrm{~g} / \mathrm{cm}^{3}\right)$. The Atwood number is $A=\left(\rho_{2}-\rho_{1}\right) /\left(\rho_{2}+\rho_{1}\right) \sim-.86$. The shock originates in the ablator and couples to the tamper while exciting perturbations imposed at the ablator/tamper interface. The shock characteristics (speed and compression) are obtained from streaked side-on radiographs as shown in Fig.1 taken with a smooth interface. For the single mode instability experiments, the perturbations are 2D and the foam is opaque while the Be ablator is transparent. The growth of the pertubations is observed directly with $2 D$ gated radiographs while a continuous record in time is obtained with streaked face-on radiographs. Examples of both are shown in Fig. 1.

Figure 2 shows the temporal evolution of the imposed mode and images from the CALE simulation for a HIGH drive case. The initial perturbation has a wavelength of $\lambda=100 \mu \mathrm{m}$ and an amplitude of $\eta_{0}=10 \mu \mathrm{m}$. The points are experimental: solid from face-on radiography and open from side-on radiography. The lines are from the simulations: solid from simulated face-on radiography and dashed from simulated side-on images. The experiment and simulation are in reasonable agreement. The perturbations invert when the shock traverses the interface near $t_{i} \sim 2 \mathrm{~ns}$ because $A<0$. They then grow at an initial rate of $d \eta / d t \sim-8 \mu \mathrm{m} / \mathrm{ns}$. At large amplitude, the growth rate declines as observed in previous experiments and simulations $[14,15]$. The simulation images show the phase inversion, shock modulation and eventual nonlinear distortion of the perturbation. 


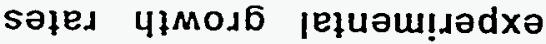

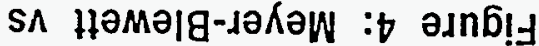

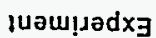

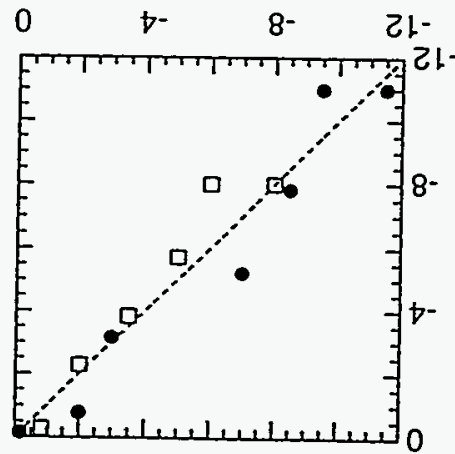

(su/uri) soles 4נmosg

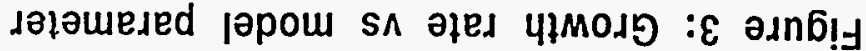

o $น \cap$ म $\forall$

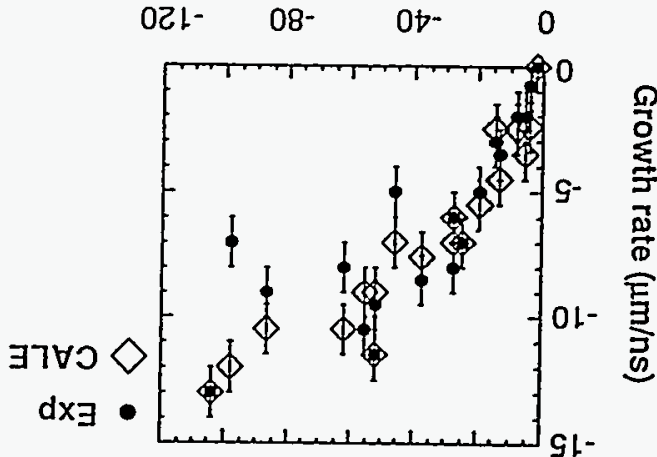

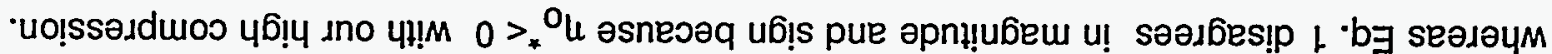

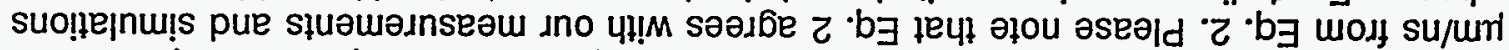

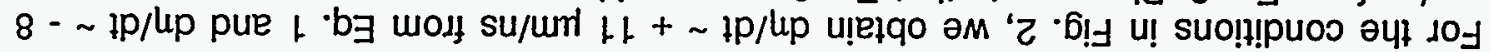

$$
Z /\left({ }_{*}^{\circ} u+O_{u}\right) \cap Y * \forall=1 p / u p
$$

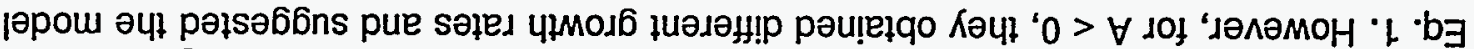

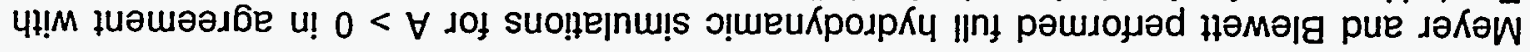

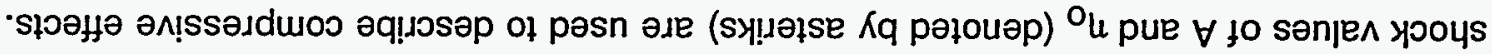

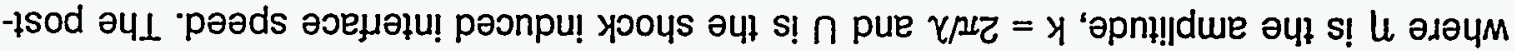

$$
{ }_{*}^{\mathrm{O}} \mathrm{L} \cap{ }_{*} \forall=\text { Ip/lup }
$$

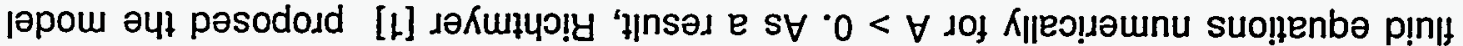

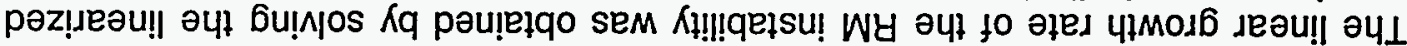

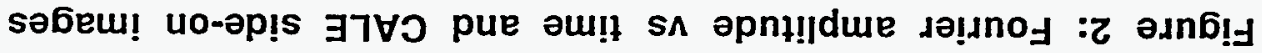
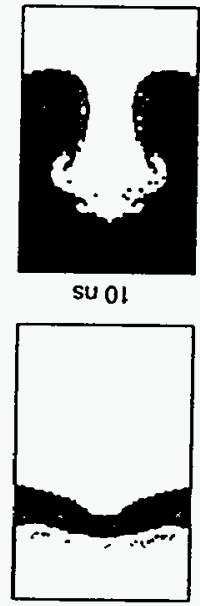

suoc

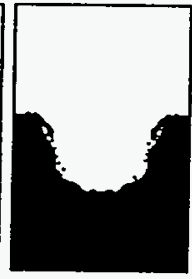

su $0 \%$

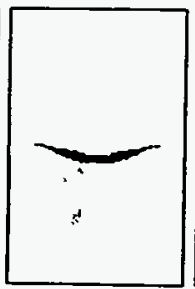

su $2 Z$
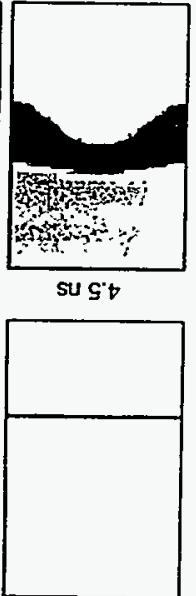

su $<1$ (su) วய!1.

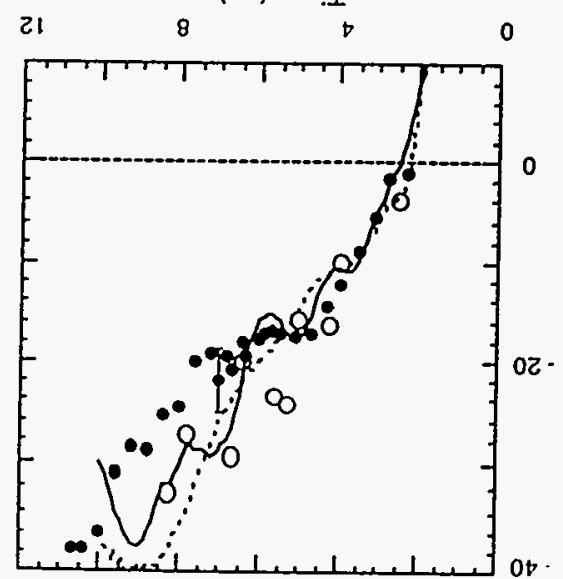

(uii) әpn!̣]due رə!ıno 
We compare the experimental and simulation growth rates over a wide range of conditions in Fig. 3 by plotting them versus the figure of merit implied by Eqs. 1 and 2 . For small values of $A k U \eta_{0}$, the growth rate increases with AkU $\eta_{0}$ consistent with the models. For large values, the growth rate approaches a limiting value perhaps due to nonlinearities. We believe the limiting value is the difference between the transmitted shock speed $V_{2}$ and the interface speed, namely, dn/dt $<V_{2}-U \sim 20 \mu \mathrm{m} / \mathrm{ns}$. This suggests that the tip of the pertubation must remain behind the transmitted shock. This seems reasonable because the pertubation spike would experience enhanced drag if it tried to penetrate the stationary upstream material.

We compare the measured growth rates with Eq. 2 in Fig. 4 for the data subset with small initial amplitude $\mathrm{k} \eta_{0}<1$. The agreement is very good. On the other hand, Eq. 1 predicts positive growth rates. These results indicate that a different expression for the growth rate is needed depending on the sign of $A$.
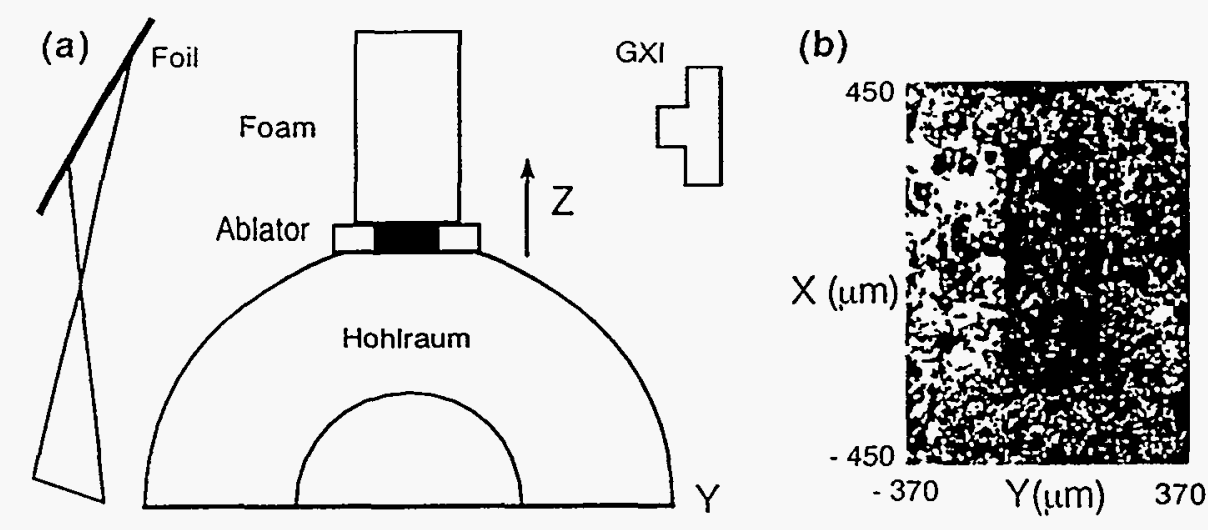

\section{Figure 5: (a) Configuration and (b) ablator for turbulence experiments}

Turbulent mix is investigated by replacing the single mode interfacial perturbations with $3 D$ random perturbations. In order to facilitate radiographic diagnosis, we modified the target because the interface can tilt or become bowed late in time and this can confuse the diagnosis. The configuration for these experiments is shown in Fig. 5. The hohlraum geometry is similar to Fig. 1, but the ablator is different as indicated by the photograph of the interface in Fig. 5b. The ablator is still berylium, but with a $250 \mu \mathrm{m}$ wide rectangular hole in the center which is filled with a Halar plastic $\left(\mathrm{C}_{4} \mathrm{H}_{4} \mathrm{~F}_{3} \mathrm{Cl}\right)$. Halar has a density $1.68 \mathrm{~g} / \mathrm{cc}$ which is similar to berylium, but contrary to $\mathrm{Be}$, it is opaque to diagnostic $x$-rays. Thus, side-on radiographs sample only the central region of the target where the tilt and curvature are minimal and the turbulent mixing zone can be obtained from $x$-ray transmission profiles. Further details are described in ref. . The imposed interfacial perturbations are 3D, random and have an root-mean-squared amplitude of $\eta_{\text {rms }} \sim 4.6 \mu \mathrm{m}$ and a peak-to-valley amplitude of $\mathrm{h} \sim 16 \mu \mathrm{m}$.

The shock-induced motion is obtained from side-on radiographs as shown in Fig 6a-c at $t=0,4.7$, and 10.2 ns. The radiographs are analyzed using the respective $x$-ray transmission profiles $T_{x r}(z)$ in Fig. $6 d-f$, which are taken near $x=0$ to minimize curvature effects and averaged over $\delta x \sim 150 \mu \mathrm{m}$ to reduce noise. The minimum in $T_{X r}(z)$ is $\sim 5 \%$ rather than the expected $0.7 \%$ for Halar due to noise. However, the ablation front $(\mathrm{A} B \mathrm{~b})$, interface $(\mathrm{I})$ and shock $(\mathrm{S})$ are discernable. At $t=0$, the target is free standing to exhibit the ablation front. At $t=4.7 \mathrm{~ns}$, the target is compressed and the transmitted shock is just ahead of the interface. At 10.2 ns, the transmitted shock is well 
ahead of the interface and the shocked foam has $T_{X r} \sim 0.6$ as expected. The interface and ablation front broaden in time. Although it is difficult to see in Fig. 6c, the shock develops an $850 \mu \mathrm{m}$ radius of curvature by $10.2 \mathrm{~ns}$, which translates to an apparent width of only $\delta \mathrm{z} \sim 9 \mu \mathrm{m}$ over the central $\delta \mathrm{x}= \pm 125 \mu \mathrm{m}$. From many measurements, we find that the incident shock reaches the interface $(z=120 \mu \mathrm{m})$ at $t_{i} \sim 3.5-4 \mathrm{~ns}$, the transmitted shock has a velocity $V_{2} \sim 70 \mu \mathrm{m} / \mathrm{ns}$ and the shock induced interface speed is $U \sim 46$ $\mu \mathrm{m} / \mathrm{ns}$. From the axial compression in the radiographs, we estimate a post-shock density of $\rho_{1}{ }^{\star} \sim 2 \mathrm{~g} / \mathrm{cm}^{3}$ for the ablator. and $\rho_{2}{ }^{\star} \sim 0.35 \mathrm{~g} / \mathrm{cm}^{3}$ for the tamper. The post-shock Atwood number is $A^{*} \sim-0.7$.

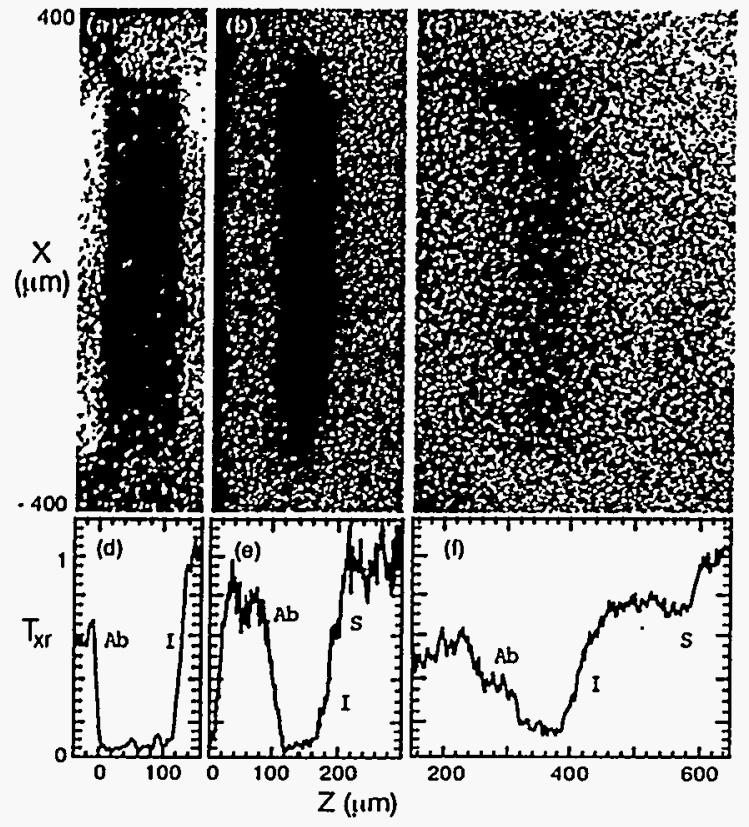

Figure 6: Nova experiment

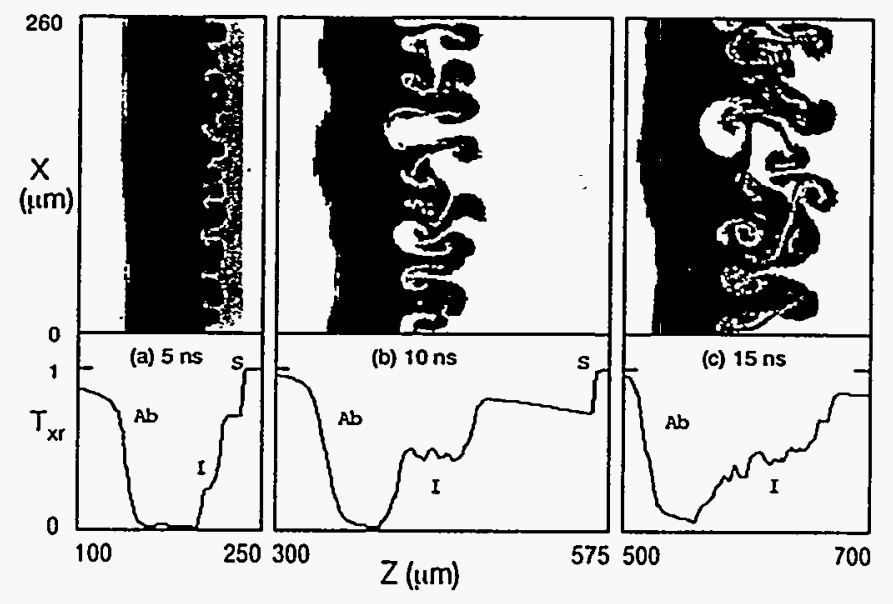

Figure 7: CALE simulation

The perturbation amplitude is determined from the gradient of the $x$-ray transmission profiles at the interface. The peak-to-valley interfacial mixing zone is identified as $h \sim \Delta T_{x r}\left(d T_{x r} / d z\right)^{-1}$, where $\Delta T_{x r}$ and $d T_{x r} / d z$ are the jump and gradient in the $x$-ray transmission at the interface, respectively. The technique is evaluated in-situ by radiographing a $50 \mu \mathrm{m}$ diameter tungsten wire and targets with known perturbations. The wire $(\tau \sim 50)$ produces a minimum transmission of $5 \%\left(\Delta \mathrm{T}_{\mathrm{Xr}}\right.$ $\sim 95 \%$ ) which is larger than expected and represents a noise baseline. In addition, the gradient length at the wire edge is $h \sim 10-12 \mu \mathrm{m}$ which is consistent with the GXI resolution. The initial profile in Fig. $6 d$ has a width of $h_{\exp } \sim 19 \mu \mathrm{m}$ at the interface where $h_{o} \sim 16 \mu \mathrm{m}$ and a smaller $h_{\exp } \sim 13 \mu \mathrm{m}$ at the ablation front where $h_{0} \sim 1-2 \mu \mathrm{m}$. We remove the systematic instrumental broadening by subtracting the GXI pinhole

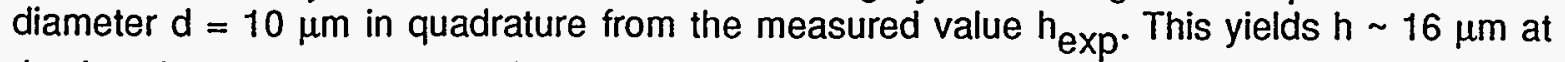
the interface in agreement with profilometry, but it still overestimates the width of the ablation front by $\sim 8 \mu \mathrm{m}$. This is a reasonable error estimate based on target tilt, 
curvature, and motion blurring. The profiles at 4.7 and $10.2 \mathrm{~ns}$ indicate a turbulent mix width of $\mathrm{h} \sim 21$ and $75 \mu \mathrm{m}$, respectively.

The experiments are compared with $2 \mathrm{D}$ hydrodynamic simulations using the Langrangian-Eulerian code CALE, which is driven with the measured radiation temperature profile. The equations of state for $\mathrm{Be}$ and Halar are approximated with $\mathrm{B}_{4} \mathrm{C}$ with density $\rho_{1}=1.65 \mathrm{~g} / \mathrm{cm}^{3}$ and the foam is represented by $\mathrm{CH}$ with $\rho_{2} \sim 0.12 \mathrm{~g} / \mathrm{cm}^{3}$. CALE obtains a peak pressure of 20 Mbar and average'velocities $U \sim 46 \mu \mathrm{m} / \mathrm{ns}$ and $\cdot V_{2} \sim$ $70 \mu \mathrm{m} / \mathrm{ns}$ similar to those measured. The incident shock velocity is $v_{1} \sim 35 \mu \mathrm{m} / \mathrm{ns}$ which has not yet been measured. Simulated side-on radiographs and associated transmission. profiles are shown in Fig. 7. The mix width increases to $h \sim 23,82$, and $113 \mu \mathrm{m}$ at 5,10, and $15 \mathrm{~ns}$. Although it is qualitative, bubble merger [13] is evident as the number of prominent features (amplitude $>.7 \mathrm{~h}$ ) declines from 12 to 8 to 4 in time. The ablation front is also distorted mainly at long wavelengths. The transmission profiles are monotonic at the interface similar to those measured except for the plateau at $10 \mathrm{~ns}$. This is not seen experimentally because the perturbations are $3 \mathrm{D}$ and the $\mathrm{x}$-rays propagate through an ensemble of spikes.

Figure 8 shows the evolution of $h$ for driven targets with $\eta_{r m s} \sim 4.6 \mu \mathrm{m}$. The points are experimental with the instrumental correction and the solid line is from 2D CALE simulations. The perturbations are compressed with a phase reversal $(A<0)$ as the shock traverses the interface between $t_{j} \sim 3$ to $4 \mathrm{~ns}$. The mix width then grows with comparable magnitudes in the experiment and simulations but at slightly different rates: To test self similarity, we fit the power law $h \sim\left(t-t_{i}\right)^{\beta}$ to the experimental data and obtain $\beta \sim 0.6$ \pm 0.1 . This agrees with the calculations [16] where $\beta \sim 0.4$ for bubbles and $\beta \sim 0.6$ for spikes at $\left|A^{\star}\right|=0.7$ (if they are symmetric in $A^{*}$ since only $0<A^{*}<1$ was considered). The CALE growth rate is nearly constant for $t<10$ ns and then declines, implying an effective $\beta<1$. This discrepancy may be due to small scale resolution limitations or that CALE is $2 \mathrm{D}$ instead of $3 \mathrm{D}$. Clearly, further developments in simulations are required.

Figure 8: Mix width vs time from experiment (points), CALE simulation (solid line), and dynamic scale model (dashed line)

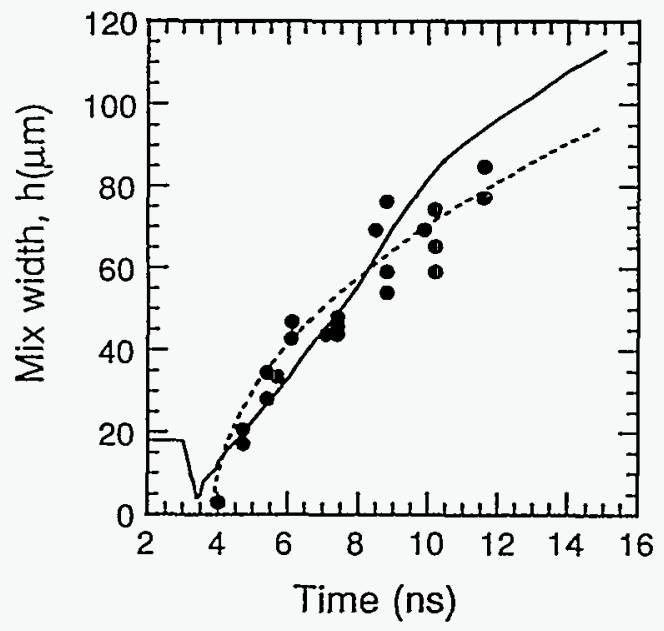

The dashed line is obtained by assuming that the peak in the turbulent wavenumber spectrum evolves in time as the amplitude increases such that $<k h>\sim$ constant. By applying this dynamic scale approximation to Eq. 2, we obtain a mix width that increases as $h \sim\left(t-t_{i}\right)^{0.5}$ consistent with the measurements. These experiments extend shock tube experiments [17] to stronger shocks and indicate the need for $3 D$ simulations[18]. 


\section{RT experiments on the LEM}

The Nova experiments are unique because they utilize a radiation drive to generate very strong shocks and high compression. However, the targets are small and turbulence is difficult to characterize over a wide range of spatial scales. Thus, we built a linear electric motor (LEM) to accelerate large fluids for diagnostic clarity and with an arbitrary acceleration profile. The configuration is shown in Fig. 9 and described in ref. [19].

The LEM consists of linear electrodes that conduct current through a sliding armature. This current and that flowing through an augmentaion coil produce a magnetic field as shown in Fig. 9. The product of the rail current and the magnetic field produces a downward force that can be programmed by varying the two current pulse shapes. The power is supplied by 16 independent circuits from an electrolytic capacitor bank with a total of 5.6 farad at 450 volt. Photographs of the LEM are shown in Fig. 10 with a long augmentaion coil and a mechanical brake. The fluid cell is placed below the armatures as seen in Fig. 10b in the accelerator and in the brake Fig. 10c. The cell has a total mass of $\sim 2 \mathrm{~kg}$ and typical fluid dimensions of $\sim 10 \mathrm{~cm}$.

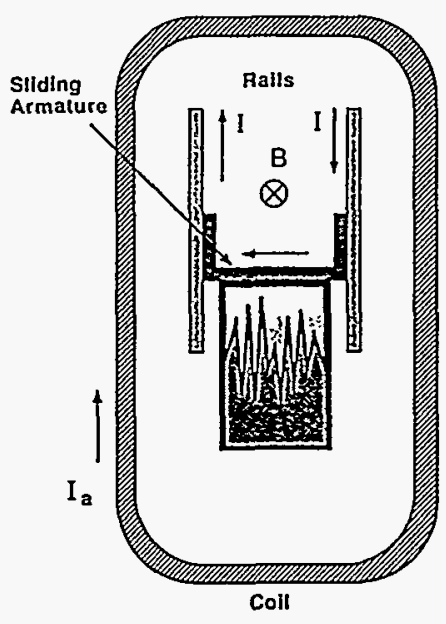

Figure 9: LEM configuration

Two representative acceleration profiles are shown in Fig. 11. A constant profile with $\mathrm{g} \sim 70 \mathrm{~g}_{\mathrm{O}}$ is obtained for $\sim 45$ ms with 12 and 20 kamp in the rail and coil circuits, respectively. After $70 \mathrm{~ms}$, the cell enters a mechanical brake which is open for diagnostic access. An impulsive profile with peak $\mathrm{g} \sim 700 \mathrm{~g}_{0}$ is obtained for $10 \mathrm{~ms}$ by increasing the rail and coil currents to 28 and 54 kamps, respectively. These profiles are designed to produce the same maximum velocity of $30 \mathrm{~m} / \mathrm{s}$. Sample shadowgraphs are shown below each profile at the same displacement $z=80 \mathrm{~cm}$ for $A=0.22$ (Freon/water). The turbulent mixing zone is the dark region in the center and clearly shows the importance of the acceleration profile. The dark region at the bottom of the impulsively accelerated case is due to cavitation in the Freon because the pressure drop accross the Freon exceeds 15 psi for $\mathrm{g}>150 \mathrm{~g}_{\mathrm{o}}$.

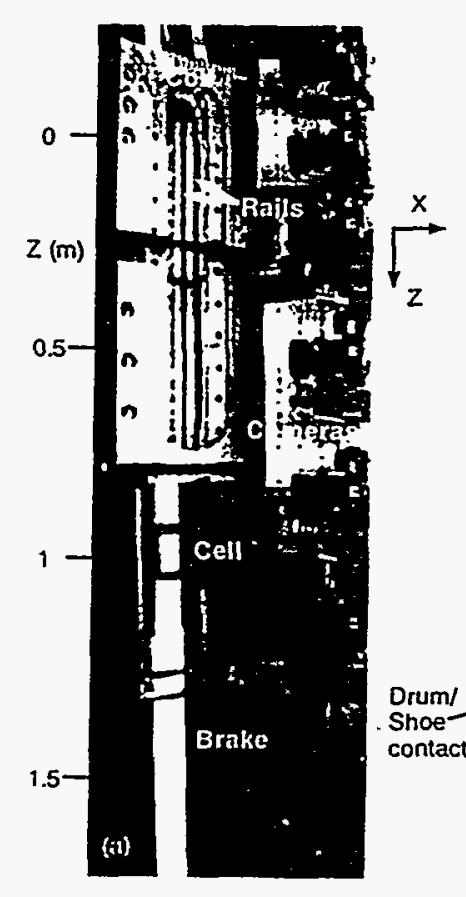

(b) Cell in accelerator

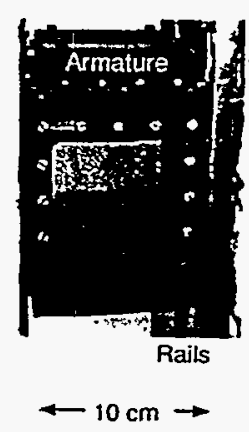

(c) Cell in brake

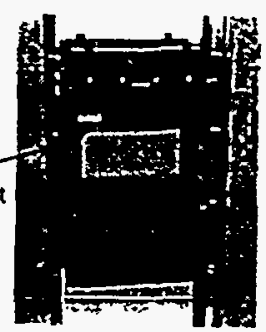

Figure 10: LEM photographs 
Constant $\mathrm{g}$

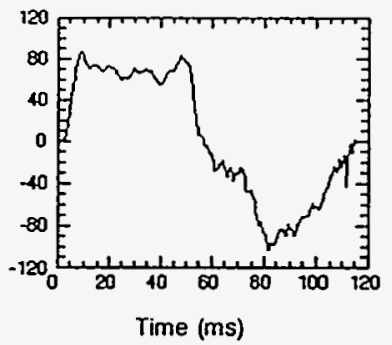

profile and shadowgraph for constant and impulsive cases
We thank the many talented people that have contributed to this work. This work was performed under the auspices of the U.S. Department of Energy by the Lawrence Livermore National Laboratory under Contract No. W-7405-ENG-48.

\section{References}

[1] R. D. Richtmyer, Commun. Pure Appl. Math 13, 297 (1960).

[2] E. E. Meshkov, Izv. Akad. Nauk SSSR Mekh. Zhidk. Gaza 4, 151 (1969) [lzv. Acad. Sci. USSR Fluid Dynamics 4, 101 (1969)].

[3] Lord Rayleigh, Scientific Papers II, 200, Cambridge, England, 1900. Sir Geoffrey

Taylor, Proc. Roy. Soc. London A201, 192 (1950).

[4] J.D. Kilkenny et al., Phys. Plasmas 1, 1379 (1994).

[5] R. Tipton (private communication).

[6] Guy Dimonte and Bruce Remington, Phys. Rev. Lett. 70, 1806 (1993).

[7] Guy Dimonte et al., (submitted to Phys. Plasmas)

[8] Guy Dimonte, Eric Frerking, and Marilyn Schneider, Phys. Rev. Lett. 74, 4855 (1995).

[9] R. F. Benjamin, D. C. Besnard, and J.F. Haas, Los Alamos Report LA-UR 92-1185.

[10] A. N. Aleshin et al., Sov. Phys. Dokl. 35, 159 (1990).

[11] M. Brouillette and B. Sturtevant, J. Fluid Mech. 263, 271 (1994).

[12] K. A. Meyer and P. J. Blewett, Phys. Fluids 15, 753 (1972).

[13] D. L. Youngs, Physica D12, 32 (1984). K.I. Read, Physica D12, 45 (1984).

[14] L. D. Cloutman and M. F. Wehner, Phys. Fluids A 4, 1821 (1992).

[15] J.W. Grove, et al., Phys. Rev. Lett. 71, 3473 (1993).

[16] U. Alon, et al., Phys. Rev. Lett. 74, 534 (1995).

[17] V. A. Andronov et al., Sov. Phys. Dokl. 27, 393 (1982). S. G. Zaitsev et al., Sov. Phys. Dokl. 30, 579 (1985).

[18] D.L. Youngs, Phys. Fluids A 3, 1312 (1991).

[19] Guy Dimonte, et al., submitted to Rev. Sci. Instruments. 